\title{
METODOLOGÍA PARA EVALUAR EL NIVEL DE PROTECCIÓN RESPIRATORIA DE MASCARILLAS Y RESPIRADORES ANTE PARTÍCULAS SIMILARES A LAS QUE TRANSMITEN EL SARS-COV-2
}

\author{
Manuel Chavez-Ruiz (iD) 1,a , Lenin Rueda-Torres (iD)1,b, Betsabé Ruffner-Camargo (D),a, \\ Cristofer Bellido-Achahui(iD) $1, \mathrm{c}$ \\ ${ }^{1}$ Centro Nacional de Salud Ocupacional y Protección del Ambiente para la Salud, Instituto Nacional de Salud, Lima, Perú. \\ ${ }^{\mathrm{a}}$ Químico, magíster en Salud Pública; ${ }^{\mathrm{b}}$ tecnólogo médico; ${ }^{\mathrm{c}}$ químico.
}

\section{RESUMEN}

Objetivo: Desarrollar una metodología para evaluar el nivel de protección respiratoria de respiradores, mascarillas quirúrgicas y mascarillas comunitarias que usa la población peruana, usando partículas de un tamaño similar a las que contienen al virus activo del SARS-CoV-2. Materiales y métodos: Se ha determinado una relación lineal directa entre el logaritmo de la concentración de partículas suspendidas en aire y el tiempo transcurrido; por lo cual es posible comparar la cantidad de partículas internas y externas a la mascarilla o respirador en un mismo periodo y conocer el porcentaje de protección respiratoria de cada muestra evaluada. Resultados: Se ha logrado implementar una metodología para evaluar el nivel de protección respiratoria ante aerosoles menores a 5,0 $\mu \mathrm{m}$. Asimismo, el empleo de accesorios como ligas o ajustadores detrás de cabeza y nuca, y el uso de clips nasales robustos, incrementan significativamente el nivel de protección respiratoria ante partículas con alta probabilidad de contener al SARS-CoV-2. Conclusiones: Se observa una concordancia entre los valores de protección respiratoria obtenidos y los esperados, considerando el nivel de filtración del material empleado de cada mascarilla quirúrgica o respirador, y su nivel de ajuste. Se observó un incremento significativo en los niveles de protección respiratoria.

Palabras clave: Respirador; Mascarillas; Aerosoles; SARS-CoV-2; Transmisión; COVID-19; Pandemia; Dispositivos de Protección Respiratoria; Filtración; Tiempo de Exposición (fuente: DeCS BIREME).

Citar como: Chavez-Ruiz M, Rueda-Torres L, Ruffner-Camargo B, Bellido-Achahui C. Metodología para evaluar el nivel de protección respiratoria de mascarillas y respiradores ante partículas similares a las que transmiten el SARS-CoV-2. Rev Peru Med Exp Salud Publica. 2021;38(3):3918. doi: https://doi.org/10.17843/ rpmesp.2021.383.8036.

Correspondencia: Manuel Chavez Ruiz; Avenida Defensores del Morro 2268, Chorrillos, Lima, Perú; mchavezr@ins.gob.pe

Recibido: 06/05/2021 Aprobado: 08/09/2021 En línea: 31/09/2021

\section{METHODOLOGY FOR EVALUATING THE LEVEL OF RESPIRATORY PROTECTION OF MASKS AND RESPIRATORS AGAINST PARTICLES SIMILAR TO THOSE THAT TRANSMIT SARS-COV-2}

\section{ABSTRACT}

Objective: To develop a methodology for evaluating the level of respiratory protection provided by respirators, surgical masks and community face masks used by the Peruvian population; protection was evaluated against particles of a size similar to those containing active SARS-CoV-2 virus. Materials and methods: A direct linear relationship has been determined between the logarithm of the concentration of airborne particles and the elapsed time; thus, it is possible to compare the quantity of particles inside and outside of the mask or respirator in the same time period, as well as to obtain the percentage of respiratory protection for each evaluated sample. Results: A methodology was established to evaluate the level of respiratory protection against aerosols smaller than $5.0 \mu \mathrm{m}$. Also, the use of accessories such as garters or adjusters behind the head and neck, and the use of strong nasal clips, significantly increased the level of respiratory protection against particles with a high probability of containing SARS-CoV-2. Conclusions: We found concordance between the obtained respiratory protection values and those expected, considering the filtration level of the material used for each surgical mask or respirator, as well as the tightness. A significant increase in the levels of respiratory protection was observed.

Keywords: Respirator; Mask; Aerosols; SARS-CoV-2; Transmition; COVID-19; Pandemic; Respiratory Protection Devices; Filtration, Exposure Time (source: MeSH NLM). 


\section{INTRODUCCIÓN}

La actual pandemia del nuevo coronavirus SARS-CoV-2, causante del síndrome agudo de distrés respiratorio ${ }^{(1)}$ continúa diezmando a comunidades enteras en todo el mundo, desafiando la capacidad de los sistemas de salud y amenazando la estabilidad económica ${ }^{(2)}$.

La principal vía de transmisión del SARS-CoV-2 son los aerosoles o microgotas respiratorias ${ }^{(3)}$. Estudios de simulación y modelado de las microgotas expelidas al hablar, toser o estornudar muestran una amplia variedad de tamaños ${ }^{(4)}$; no obstante, se estima que más del $90 \%$ se encuentra en forma de aerosoles, menores a $5 \mu \mathrm{m}$ de diámetro ${ }^{(5,6)}$. Estas partículas pueden permanecer suspendidas en el aire de ambientes sin ventilación por tres horas ${ }^{(7)}$. Otro estudio reporta un tiempo de suspensión de hasta por 16 horas ${ }^{(8,9)}$, lo cual favorece la probabilidad de contacto con personas circulantes.

El uso obligatorio de equipos de protección respiratoria (EPR) es una medida de prevención primaria que busca limitar la cadena de transmisión viral. Los respiradores y mascarillas certificadas limitan el paso de partículas de un gran espectro de tamaños y composición. Los respiradores N95 sustentan una eficiencia de filtración de partículas (EFP) de al menos $95 \%$ de partículas no oleosas de $0,3 \mu \mathrm{m}$ de diámetro, por lo cual se emplean principalmente en ambientes de alto riesgo biológico, como las áreas hospitalarias ${ }^{(10)}$. Sin embargo, un alto índice de EFP no está necesariamente ligado a un buen nivel de protección respiratoria (NPR) ante micropartículas, debido, en especial, a la falta de ajuste, lo cual genera puntos de fuga de aire por donde escapan e ingresan estas partículas.

A nivel comunitario circulan las mascarillas no médicas, de diversos materiales, combinaciones y diseños de confección, conforme a las directrices de cada país. A pesar de evidenciar considerablemente menor eficiencia de filtración que los respiradores $\mathrm{N} 95^{(11,12)}$, la efectividad de esta intervención en ciertas poblaciones parece contribuir positivamente a la mitigación de la transmisibilidad viral ${ }^{(13,14)}$. Bajo el contexto de las nuevas variantes del SARS-CoV-2 que advierten mayor transmisibilidad y letalidad de la infección, apremia prestar atención a medidas simples y factibles que sustenten un mayor grado de protección en el entorno comunitario.

Medidas asequibles y comprobadas son importantes estrategias contra la pandemia mientras se consiga extender el proceso de vacunación a la mayor cantidad de la población. El presente estudio propone una metodología para evaluar el nivel de protección respiratoria (NPR) de mascarillas quirúrgicas, respiradores KN95 y otras mascarillas de uso comunitario; asimismo, se evaluó la adición de accesorios y diversos tipos de usos para mejorar el nivel de ajuste de las mascarillas y, con ello, el NPR.

\section{MENSAJES CLAVE}

Motivación para realizar el estudio: Falta de metodologías disponibles para evaluar el nivel de protección respiratoria ante partículas con probable carga del SARS-CoV-2.

Principales hallazgos: Se ha encontrado que mejorar el ajuste de las mascarillas, así como el empleo de accesorios como ajustadores detrás de la cabeza y clip nasales robustos, puede incrementar significativamente el nivel de protección respiratoria.

Implicancias: Los tomadores de decisiones podrían aplicar estos hallazgos para mejorar la efectividad de los equipos de protección respiratoria contra el SARS-CoV-2.

\section{MATERIALES Y MÉTODOS}

\section{Diseño}

El presente estudio experimental se ha realizado en el Laboratorio de Evaluación de Riesgos Ocupacionales del Instituto Nacional de Salud del Perú.

\section{Variables}

El NPR se define como la cantidad de micropartículas que logran penetrar en el EPR con las partículas externas; esto se determina en mascarillas quirúrgicas, respiradores KN95 y algunas modificaciones que buscan mejorar el NPR al aumentar el ajuste. La EFP se define como un parámetro que busca determinar el aporte de la filtración del material empleado en la fabricación de los EPR en el resultado del NPR.

\section{Procedimiento}

La metodología empleada para determinar el NPR, propuesta en el presente estudio, se basa en la medición de la concentración de partículas, definida como el número de partículas por litro de aire, del ambiente externo $\left(\# \mathrm{P}_{\text {ext }}\right)$ y de aquellas que lograron penetrar el EPR $\left(\# \mathrm{P}_{\text {int }}\right)$. Para esta medición se utilizó un maniquí al cual se le agregó una lámina de silicona para mejorar la adherencia del EPR a la superficie, se utilizaron dos sondas, colocadas en la parte posterior, una para la toma de muestra de partículas que penetraron el EPR y la otra para simular la respiración humana (Figura 1), la cual se adaptó a seis litros por minuto (volumen de respiración por minuto).

Para asegurar la presencia, cantidad y tamaños de aerosoles se liberaron partículas en el ambiente donde se realizó el ensayo; para ello se utilizó un generador de partículas neumático elaborado en el Laboratorio de Química del Instituto Nacional de Salud, el cual genera partículas polidispersas a partir de una solución de cloruro de sodio al 2\%; las partí- 
culas generadas tenían 2,5 $\mu \mathrm{m}$ de diámetro. La medición se realizó cuando la cantidad de partículas en el ambiente fue significativamente mayor a la cantidad de partículas, una vez llegado el equilibrio. Para esto se empleó un equipo contador de partículas de marca PCE modelo 01L, el cual fue calibrado previamente. El lugar empleado para los ensayos tuvo un área de $1,5 \times 2,5 \mathrm{~m}$; con una humedad relativa y temperatura ambiental, entre $50-60 \%$ y $20-25{ }^{\circ} \mathrm{C}$, respectivamente. El NPR se obtuvo al relacionar el $\# \mathrm{P}_{\text {ext }}$ con el \# $\mathrm{P}_{\text {int }}$ en el mismo periodo y se expresó en porcentaje.

$$
\mathrm{NPR}=\left(1-\# \mathrm{P}_{\mathrm{int}} / \# \mathrm{P}_{\text {ext }}\right) \times 100
$$

NPR: nivel de protección respiratoria; $\# \mathrm{P}_{\text {int }}$ : número de partículas por litro de aire internas al EPR; $\# \mathrm{P}_{\text {ext }}$ : número de partículas por litro de aire del ambiente externo.

Para determinar el \# $\mathrm{P}_{\text {ext }}$, se tomaron lecturas del ambiente cada 15 segundos, se realizó un gráfico de $\# \mathrm{P}_{\text {ext }}$ versus el tiempo transcurrido (Figura 2A) donde se aprecia una relación logarítmica entre ambos. Se procedió a linealizar la gráfica de la Figura 2A con el logaritmo del \# $\mathrm{P}_{\text {ext }}$ versus el tiempo transcurrido (Figura 2B). Finalmente se obtuvo la ecuación de esta nueva relación lineal, con la cual se pudo obtener el $\# \mathrm{P}_{\text {ext }}$ en el mismo periodo donde la sonda del equipo contador de partículas tomaba lecturas en el interior del $\mathrm{EPR}$, para obtener los datos del \# $\mathrm{P}_{\text {int }}$.

$$
\log \left(\# \mathrm{P}_{\text {ext }}\right)=\mathrm{b} \times \mathrm{t}+\mathrm{a}
$$

b: pendiente de la ecuación; t: tiempo transcurrido en segundos desde el inicio del ensayo; a: constante derivada de la relación lineal.

Adicionalmente, con el objetivo de evaluar la capacidad de filtración del material con el cual están fabricados los EPR, se procedió a determinar la EFP. Para este ensayo se ha seguido el procedimiento de la National Institute for Occupational and Safety and Health (NIOSH), TEB-APRSTP-0059 ${ }^{(15)}$, cabe precisar que esta metodología analiza el EPR colocado en un portamuestra y sellado herméticamente por el contorno, eliminando el factor de fuga de aire, sin considerar el ajuste del EPR, a diferencia del método propuesto en el presente estudio.

Se evaluaron mascarillas quirúrgicas y respiradores KN95, así como el incremento del NPR al realizar modificaciones en el modo de uso y la adición de accesorios que mejoren el ajuste de los EPR (Tabla 1).

\section{Análisis estadístico}

Para determinar el NPR se realizaron tres repeticiones y para determinar la EFP se realizaron seis repeticiones. Los resultados de ambas pruebas se expresaron con la media y la desviación estándar.

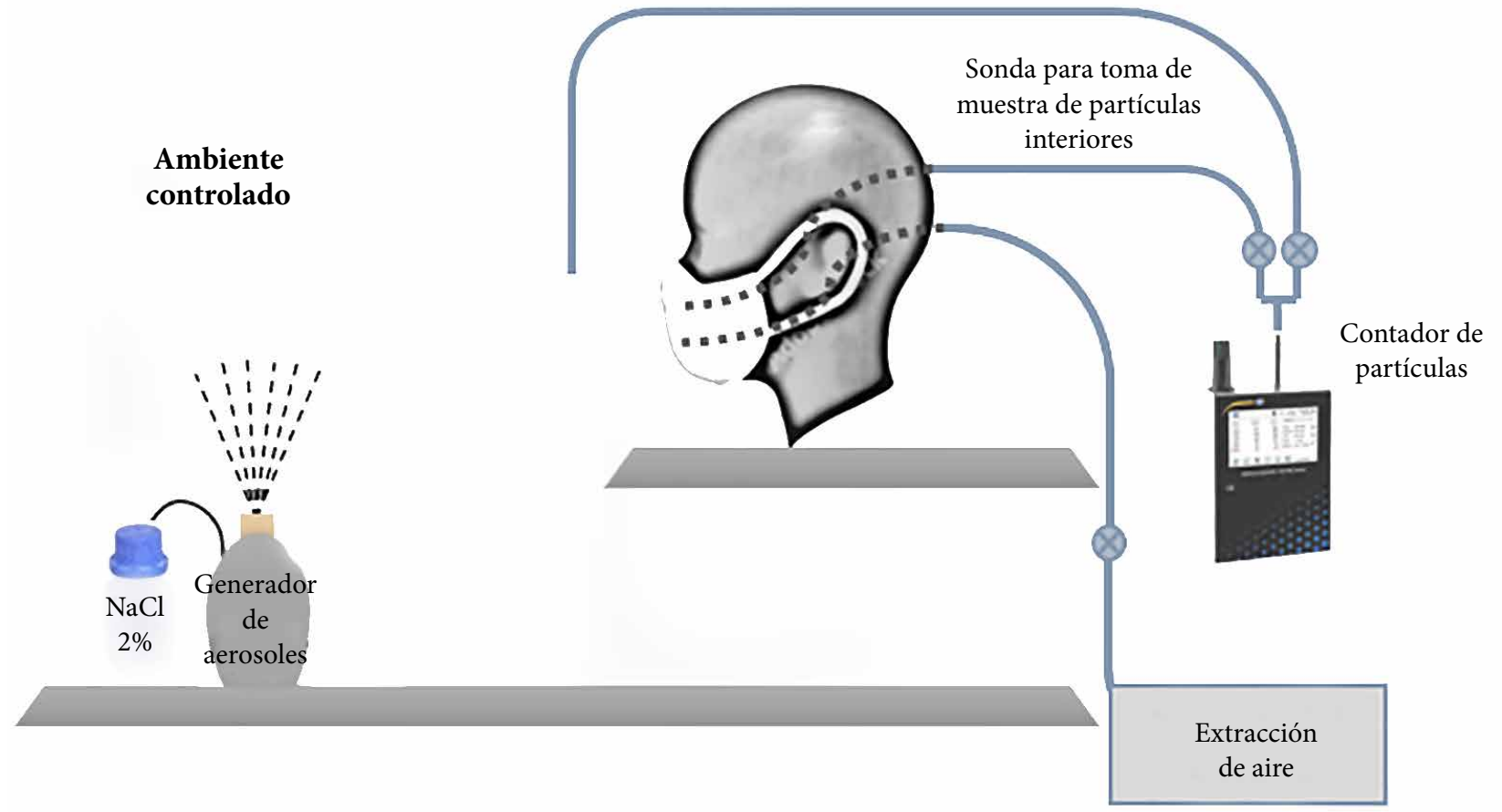

Sonda para toma de muestra de partículas del ambiente

Figura 1. Sistema de medición de partículas externas e internas al equipo de protección respiratoria para determinar el nivel de protección respiratoria. 


\section{A}

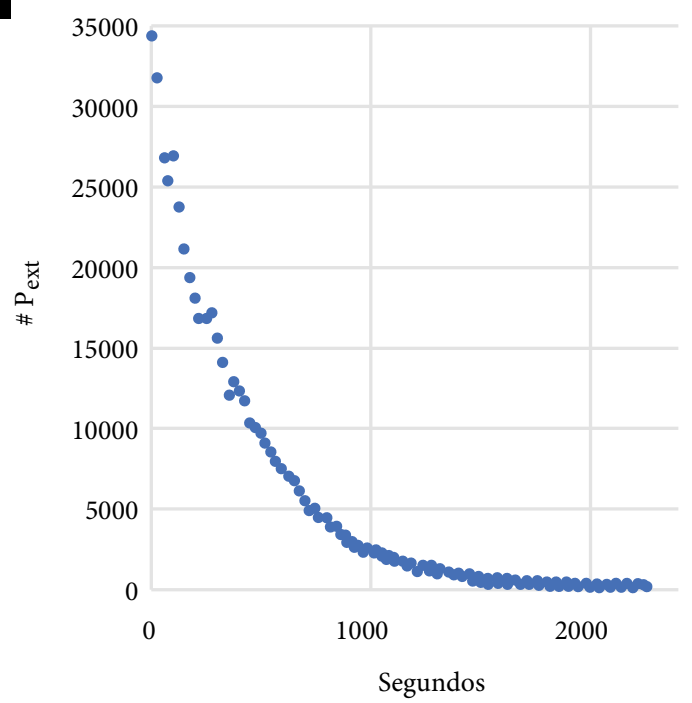

B

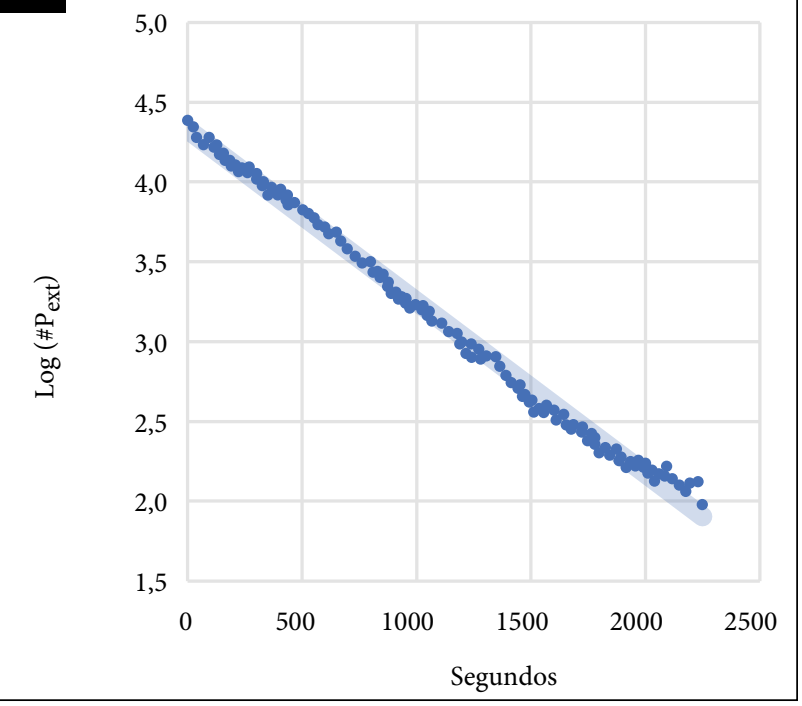

Figura 2. Comportamiento de los aerosoles con el tiempo. A) Relación del número de partículas ambientales externas por litro de aire versus el tiempo transcurrido en segundos. B) Relación del logaritmo del número de partículas ambientales externas por litro de aire versus el tiempo transcurrido en segundos.

\section{RESULTADOS}

La EFP y el NPR en la mascarilla quirúrgica fueron de $97 \%$ y $32 \%$, respectivamente; se midieron los valores del NPR en las modificaciones descritas en la Tabla 1, que dieron como resultado incrementos del NPR de acuerdo con el siguiente orden: MQ-MQ (41\%), MQ-TL (74\%), MQ-LG (66\%), MQLG-CL (91\%). La EFP y el NPR del respirador KN95 fueron de $99 \%$ y $75 \%$, respectivamente. Asimismo, los NPR de las modificaciones planteadas en la Tabla 1 dieron como resultado un incremento del NPR con el siguiente orden: MQKN95 (89\%) y KN95-LG (97\%) (Tabla 2, Figura 3).

Tabla 1. Descripción de las modificaciones y combinaciones probadas para determinar los niveles de protección respiratoria de los equipos de protección de uso común en la población.
Tipo de protección
Modificación
Código
Comentario

\begin{tabular}{|c|c|c|c|}
\hline \multirow{5}{*}{ Mascarilla quirúrgica } & Sin modificación & MQ & $\begin{array}{l}\text { Mascarilla quirúrgica marca R\&G, lote } 2732220301 \text {, de tres } \\
\text { capas con clip nasal débil y ligas de sujeción a las orejas }\end{array}$ \\
\hline & $\begin{array}{l}\text { Adición de una segunda mascarilla } \\
\text { quirúrgica }\end{array}$ & MQ-MQ & $\begin{array}{l}\text { Dos mascarillas quirúrgicas puestas una encima de otra con } \\
\text { sujeción a las orejas }\end{array}$ \\
\hline & $\begin{array}{l}\text { Adición de liga elastomérica para } \\
\text { ajuste a la cabeza }\end{array}$ & MQ-LG & Inserción de liga elastomérica para ajuste a la cabeza. \\
\hline & $\begin{array}{l}\text { Adición de segunda mascarilla de } \\
\text { tela encima }\end{array}$ & MQ-TL & $\begin{array}{l}\text { Adición de mascarilla de tela con ajuste a la cabeza y clip nasal } \\
\text { de aluminio robusto }\end{array}$ \\
\hline & $\begin{array}{l}\text { Adición de liga elastomérica para } \\
\text { ajuste a la cabeza y clip nasal ro- } \\
\text { busto }\end{array}$ & MQ-LG-CL & $\begin{array}{c}\text { Inserción de liga elastomérica para ajuste a la cabeza y clip } \\
\text { nasal de aluminio robusto }\end{array}$ \\
\hline \multirow{3}{*}{ Respirador KN95 } & Sin modificación & KN95 & $\begin{array}{c}\text { Respirador KN95 marca Wenzhou Kanghong, lote } \\
\text { Hy20200501, con sujetador a las orejas y clip nasal metálico } \\
\text { medianamente robusto }\end{array}$ \\
\hline & $\begin{array}{l}\text { Colocación de una mascarilla qui- } \\
\text { rúrgica debajo del respirador KN95 }\end{array}$ & MQ-KN95 & $\begin{array}{l}\text { Mascarilla quirúrgica sujetada a las orejas y encima respirador } \\
\qquad \text { KN95 }\end{array}$ \\
\hline & $\begin{array}{c}\text { Adición de liga elastomérica para } \\
\text { ajuste a cabeza }\end{array}$ & KN95-LG & $\begin{array}{c}\text { Respirador KN95 al cual se le adicionó una liga elastomérica } \\
\text { para ajuste a la cabeza }\end{array}$ \\
\hline $\begin{array}{l}\text { Respirador N95 } \\
\text { certificado }\end{array}$ & Sin modificación & N95 & $\begin{array}{l}\text { Respirador N95 marca } 3 \mathrm{M} \text {, lote } 8060 \text {, certificado por NIOSH, } \\
\text { ligas de sujeción a cabeza, clip nasal de aluminio robusto }\end{array}$ \\
\hline
\end{tabular}


Tabla 2. Resultados de la eficiencia de filtración de partículas y del nivel de protección respiratoria de los equipos de protección y sus modificaciones

\begin{tabular}{lcc}
\hline Código & $\begin{array}{c}\text { EFP (\%) } \\
\text { Media (DE) }\end{array}$ & $\begin{array}{c}\text { NPR (\%) } \\
\text { Media (DE) }\end{array}$ \\
\hline MQ & $97(0,8)$ & $32(10)$ \\
MQ-MQ & - & $41(0,5)$ \\
MQ-LG & - & $66(3,9)$ \\
MQ-TL & - & $74(4,6)$ \\
KN95 & $99(0,1)$ & $75(4,7)$ \\
MQ-KN95 & - & $89(2,2)$ \\
MQ-LG-CL & - & $91(1,7)$ \\
KN95-LG & - & $97(1,2)$ \\
N95 & $99(0,2)$ & $99,6(0,1)$ \\
\hline
\end{tabular}

EFP: eficiencia de filtración de partículas; NPR: nivel de protección respiratoria; DE: desviación estándar; MQ: mascarilla quirúrgica; MQ-MQ: dos mascarillas quirúrgicas; MQ-LG: mascarilla quirúrgica con inserción de liga elastomérica; MQ-TL: mascarilla quirúrgica con adición de una mascarilla de tela con ajuste a la cabeza y clip nasal de aluminio robusto; KN95: respirador KN95; MQ-KN95: mascarilla quirúrgica y respirador KN95; MQ-LG-CL: mascarilla quirúrgica con inserción de liga elastomérica para ajuste a la cabeza y clip nasal de aluminio robusto; KN95-LG: respirador KN95 con adición una liga elastomérica; N95: respirador N95.

\section{DISCUSIÓN}

Se aprecia una alta EFP en ambos dispositivos evaluados, $99 \%$ para el respirador KN95 y 97\% para la mascarilla quirúrgica. Sin embargo, el NPR para ambos dispositivos (75\% y $32 \%$, respectivamente) refleja problemas de ajuste, lo que demuestra que el NPR no depende solo de la EFP del material de los EPR. Por otro lado, existen algunas alternativas que mejoran significativamente el ajuste y, por ende, el NPR.

La metodología propuesta en el presente trabajo se basa en la medición de las partículas que lograron sobrepasar el EPR, a diferencia del método Fit Testing Procedure, indicado por la Occupational Safety \& Health Administration (OSHA) ${ }^{(16)}$, donde se emplea un detector, basado en el principio de condensación, el cual incluye la medición de partículas de 0,02 a $0,08 \mu \mathrm{m}$, por lo que es improbable que las mismas contengan al menos una unidad de virus activo del SARS-CoV-2, cuyo tamaño se ha estimado en $0,08+/-0,01 \mu \mathrm{m}^{(17,18)}$.

A pesar de la limitada evidencia sobre la presencia de SARS-CoV-2 activos y su distribución en partículas menores a $10 \mu \mathrm{m}$, se ha determinado que la capacidad de partículas para transportar virus activos se incrementa con el tamaño de estas, lo que podría explicarse por un efecto de mayor blindaje del virus al aumentar el tamaño de las gotas que lo hospedan ${ }^{(19)}$. Se ha descrito la presencia de virus de la influenza A (IAV) y del síndrome respiratorio y reproductivo porcino (PRRS) en partículas iguales y más grandes que $2,1 \mu \mathrm{m}^{(20)}$; mientras, en otros estudios ${ }^{(21)}$ se ha demostrado la presencia del SARS-CoV-2 en partículas menores a $10 \mu \mathrm{m}$.
En un estudio de exposición empleando el modelo murino, virus de la influenza aerosolizada en partículas de $2 \mu \mathrm{m}$, produjeron una menor infección en comparación a las partículas de mayor tamaño $(10 \mu \mathrm{m})^{(22)}$. Por otro lado, las partículas finas de 2,5 $\mu \mathrm{m}$ de diámetro aerodinámico representan un tamaño de interés para la salud debido a que son suficientemente pequeñas para penetrar hasta los pulmones y dañar la pared alveolar ${ }^{(23)}$. Por los hechos indicados, en el presente estudio se ha visto conveniente trabajar con partículas de $2,5 \mu \mathrm{m}$, por la alta probabilidad de contener virus y para realizar las mediciones de concentración de partículas ambientales e internas al dispositivo de protección respiratoria.

Los Centros para el Control y la Prevención de Enfermedades (CDC) refieren que el colocar una mascarilla de tela sobre una quirúrgica mejora significativamente la eficiencia de filtración ${ }^{(24)}$. Algunas opciones para mejorar el NPR, como el anudar las ligas de las mascarillas, uso de gasas para sellar aberturas visibles, entre otras, han demostrado mejores resultados en la protección respiratoria en relación a la forma original ${ }^{(24,25)}$.

El resultado del NPR para el respirador KN95 del 75\%, relacionado al $99 \%$ obtenido en su EFP, indica que el ajuste con sujeción a las orejas no es óptimo, por lo cual esto mejoraría con una mascarilla quirúrgica debajo del mismo (89\%). Otro aspecto a tener en cuenta es que estos EPR vienen en tallas únicas por lo cual estos resultados podrían variar de acuerdo a las características anatómicas de cada persona. El máximo NPR para este dispositivo se obtiene al adicionar una liga elastomérica con ajuste a la cabeza (NPR $=97 \%)$.

En el caso de las mascarillas quirúrgicas, en la medición del NPR (colocada tal cual se venden), se ha encontrado que, a pesar de su alta EFP (97\%), presenta un pobre NPR (32\%), esto se debería al mal ajuste de estos dispositivos, que han sido desarrollados para evitar la emisión de partículas por parte del usuario al exterior y no como un dispositivo de protección respiratoria.

En las modificaciones realizadas, los mejores resultados se obtuvieron al adicionar una liga elastomérica y clip nasal (91\%), lo cual mejoró el ajuste y el sellado de las fugas de aire que quedan en la parte superior de la nariz. Esto se comprobó cuando se midió la modificación referida al empleo de la liga de sujeción a la cabeza $(\mathrm{NPR}=66 \%)$, lo que resalta la importancia de las fugas que quedan a los lados de la nariz en la protección respiratoria. La mascarilla de tela encima incrementó el NPR (74\%); sin embargo, se debe mencionar que la mascarilla de tela que se usó tenía ligas de sujeción detrás de la cabeza y nuca, y un clip nasal, por lo cual el uso de mascarillas de tela con un mal ajuste podría arrojar valores mucho menores al encontrado en el presente estudio. El empleo de dos mascarillas quirúrgicas con ajuste a las orejas tuvo un NPR del $41 \%$ y mostró solo un ligero incremento; esta modificación mantiene la configuración básica con evidentes aberturas laterales, por lo que el CDC no recomienda su uso ${ }^{(26)}$. 


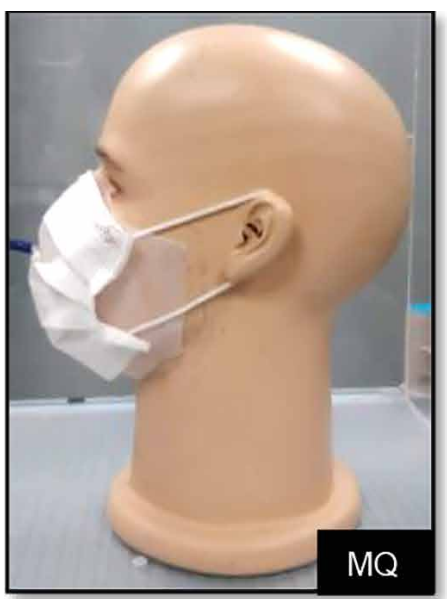

A)

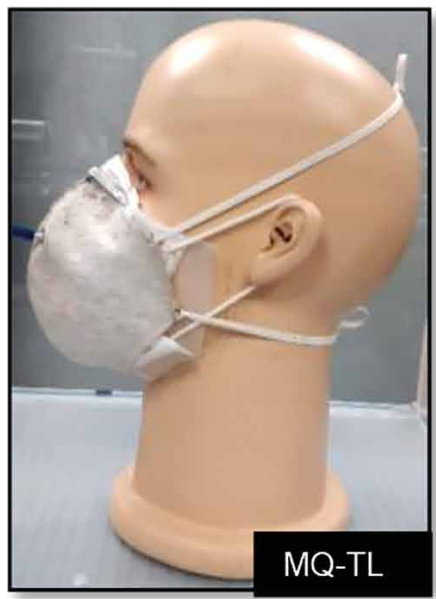

D)

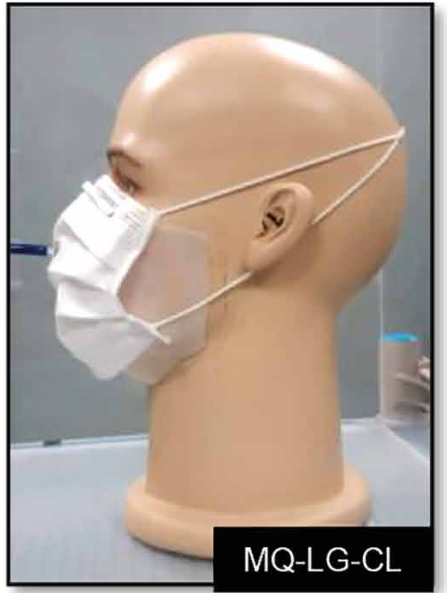

G)

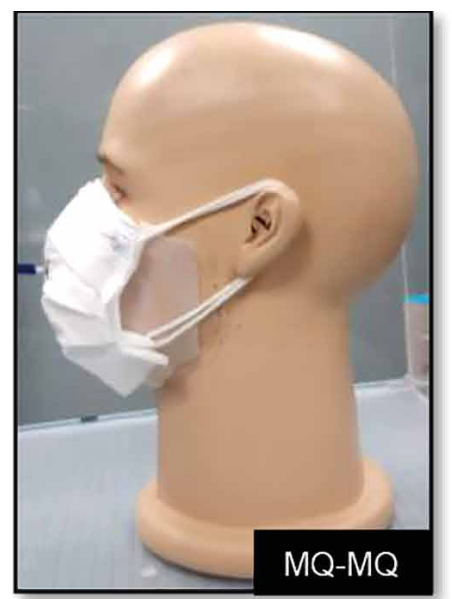

B)

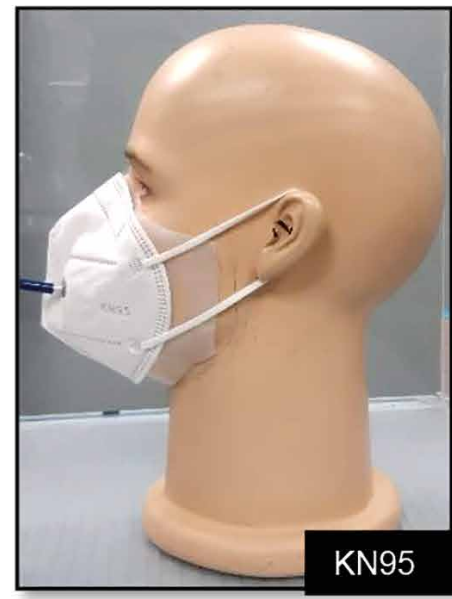

E)

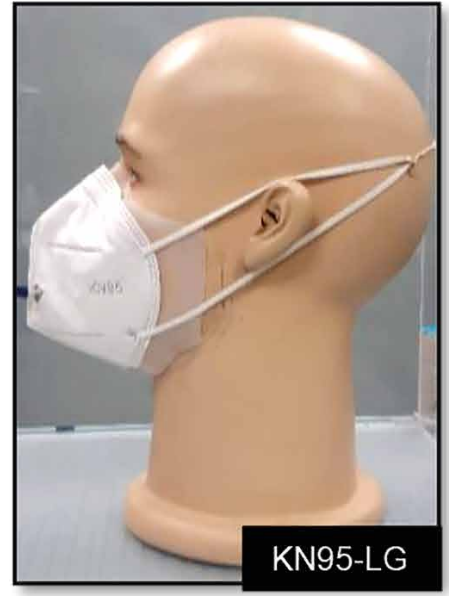

H)

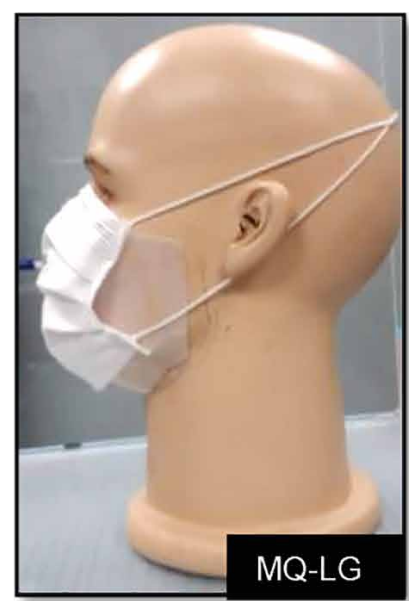

C)

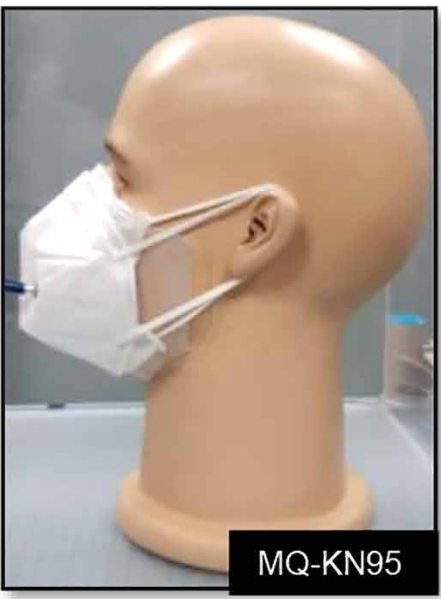

F)

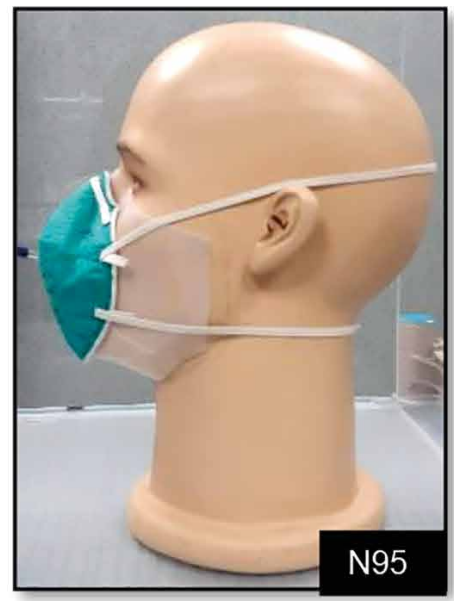

I)

Figura 3. A) MQ: mascarilla quirúrgica de tres capas con clip nasal débil sujetada a las orejas. B) MQ-MQ: dos mascarillas quirúrgicas puestas una encima de otra sujetadas a las orejas. C) MQ-LG: mascarilla quirúrgica con inserción de liga elastomérica para ajuste a la cabeza. D) MQ-TL: mascarilla quirúrgica con adición de una mascarilla de tela con ajuste a la cabeza y clip nasal de aluminio robusto. E) KN95: respirador KN95 con sujetador a las orejas y clip nasal metálico medianamente robusto. F) MQ-KN95: mascarilla quirúrgica sujetada a las orejas y encima respirador KN95. G) MQ-LGCL: mascarilla quirúrgica con inserción de liga elastomérica para ajuste a la cabeza y clip nasal de aluminio robusto. H) KN95-LG: respirador KN95 con adición de una liga elastomérica para ajuste a la cabeza. I) N95: respirador N95 certificado NIOSH con ligas a la cabeza y clip nasal de aluminio robusto. 
El valor de NPR del 99,6\% encontrado para el respirador N95 de marca 3M modelo 1860 tiene concordancia con el valor de EFP y el ajuste mostrado por dicho EPR, el cual sirve como prueba de validación de la metodología propuesta.

Nuestros hallazgos resaltan que el buen ajuste de la mascarilla es tan importante como el material de su composición. Aditamentos simples como bandas elásticas detrás de la cabeza y nuca, incrementa en buena medida los niveles de protección, y los clips nasales pueden aumentar significativamente el nivel de protección respiratoria de los EPR.

Como limitación se debe mencionar la poca evidencia científica sobre el rango de tamaños de partículas que defina el mínimo tamaño de partículas con carga del SARS-CoV-2. Asimismo, la variedad y procedencia de las mascarillas quirúrgicas y respiradores KN95 es muy amplia, por lo que la muestra utilizada en este estudio no sería representativa del universo de estos dispositivos.

En conclusión, la metodología propuesta para evaluar el NPR ante aerosoles representada por partículas finas de 2,5 $\mu \mathrm{m}$ tiene resultados concordantes con los valores de la EFP de las muestras evaluadas, y el nivel de ajuste observado. En el caso de las mascarillas quirúrgicas se demostró un pobre NPR debido al mal ajuste, y en los respiradores KN95 se obtuvo un mayor NPR; sin embargo, se ha demostrado que la modificación con el uso de ligas y clips nasales pueden mejorar considerablemente el ajuste de ambos EPR. Estos hallazgos pueden ser considerados para establecer normativas en salud pública con el objetivo de mejorar las políticas de prevención en la exposición ante aerosoles con alta probabilidad de contener el SAR-CoV-2.

\section{REFERENCIAS BIBLIOGRÁFICAS}

1. Li Q, Guan X, Wu P, Wang X, Zhou L, Tong Y, et al. Early Transmission Dynamics in Wuhan, China, of Novel Coronavirus-Infected Pneumonia. N Engl J Med. 2020;382(13):1199-207. doi: 10.1056/NEJMoa2001316.

2. Ibn-Mohammed T, Mustapha KB, Godsell J, Adamu Z, Babatunde KA, Akintade DD, et al. A critical analysis of the impacts of COVID-19 on the global economy and ecosystems and opportunities for circular economy strategies. Resour Conserv Recycl. 2021;164:105169. doi: 10.1016/j. resconrec.2020.105169.

3. Greenhalgh T, Jimenez JL, Prather KA, Tufekci Z, Fisman D, Schooley $\mathrm{R}$. Ten scientific reasons in support of airborne transmission of SARSCoV-2. Lancet. 2021;397(10285):1603-1605. doi: 10.1016/S01406736(21)00869-2.4.

4. Han ZY, Weng WG, Huang QY. Characterizations of particle size distribution of the droplets exhaled by sneeze. J R Soc Interface. 2013;10(88):20130560. doi: 10.1098/rsif.2013.0560.

5. Zayas G, Chiang MC, Wong E, MacDonald F, Lange CF, Senthilselvan $\mathrm{A}$, et al. Cough aerosol in healthy participants: fundamental knowledge to optimize droplet-spread infectious respiratory disease management. BMC Pulm Med. 2012;12:11. doi: 10.1186/1471-2466-12-11.

6. Asadi S, Wexler AS, Cappa CD, Barreda S, Bouvier NM, Ristenpart WD. Aerosol emission and superemission during human speech increase with voice loudness. Sci Rep. 2019;9(1):2348. doi: 10.1038/s41598-019-38808-z.

7. van Doremalen N, Bushmaker T, Morris DH, Holbrook MG, Gamble A, Williamson BN, et al. Aerosol and Surface Stability of SARS-CoV-2 as Compared with SARS-CoV-1. N Engl J Med. 2020;382(16):1564-1567. doi: 10.1056/NEJMc2004973.

8. Smither SJ, Eastaugh LS, Findlay JS, Lever MS. Experimental aerosol survival of SARS-CoV-2 in artificial saliva and tissue culture media at medium and high humidity. Emerg Microbes Infect. 2020;9(1):1415-1417. doi: 10.1080/22221751.2020.1777906.

9. Fears AC, Klimstra WB, Duprex P, Hartman A, Weaver SC, Plante KS, et al. Persistence of Severe Acute Respiratory Syndrome Coronavirus 2 in Aerosol Suspensions. Emerg Infect Dis. 2020;26(9):2168-2171. doi: 10.3201/eid2609.201806.

10. Whiley H, Keerthirathne TP, Nisar MA, White MAF, Ross KE. Viral Filtration Efficiency of Fabric Masks Compared with Surgical and N95 Masks. Pathogens. 2020;9(9):762. doi: 10.3390/pathogens9090762.

11. Jung H, Kim JK, Lee S, Lee J, Kim J, Tsai P, et al. Comparison of Filtration Efficiency and Pressure Drop in Anti-Yellow Sand Masks, Quarantine Masks, Medical Masks, General Masks, and Handkerchiefs. Aerosol Air
Qual Res. 2014;14(3):991-1002. doi: 10.4209/aaqr.2013.06.0201.

12. Li Y, Wong T, Chung J, Guo YP, Hu JY, Guan YT, et al. In vivo protective performance of N95 respirator and surgical facemask. Am J Ind Med. 2006;49(12):1056-65. doi: 10.1002/ajim.20395.

13. Brooks JT, Butler JC. Effectiveness of Mask Wearing to Control Community Spread of SARS-CoV-2. JAMA. 2021;325(10):998-999. doi:10.1001/ jama.2021.1505.

14. Bragazzi NL, Mahroum N, Damiani G, Kong JD, Wu J. Effectiveness of community face mask use on COVID-19 epidemiological trends and patterns in Italy: evidence from a "translational" study. Infect Dis (Lond). 2021;53(4):252-254. doi: 10.1080/23744235.2021.1883731.

15. National Institute for Occupational Safety and Health. Determination of Particulate Filter Efficiency Level for N95 Series Filters Against Solid Particulates for Non-Powered, Air-Purifying Respirators Standard Test Procedure (STP). TEB-APR-STP-0059. 2019. Disponible en: https://www. cdc.gov/niosh/npptl/stps/pdfs/TEB-APR-STP-0059-508.pdf.

16. Occupational Safety and Health Administration. Appendix A to $\$ 1910.134$ App A - Fit Testing Procedures (Mandatory). OSHA [Internet]. Disponible en: https://www.osha.gov/laws-regs/regulations/standardnumber/1910/1910.134AppA.

17. Park WB, Kwon N-J, Choi S-J, Kang CK, Choe PG, Kim JY, et al. Virus Isolation from the First Patient with SARS-CoV-2 in Korea. J Korean Med Sci. 2020;35(7):e84. doi: 10.3346/jkms.2020.35.e84.

18. Kim J-M, Chung Y-S, Jo HJ, Lee N-J, Kim MS, Woo SH, et al. Identification of Coronavirus Isolated from a Patient in Korea with COVID-19. Osong Public Health Res Perspect. 2020;11(1):3-7. doi: 10.24171/j. phrp.2020.11.1.02.

19. Zuo Z, Kuehn TH, Verma H, Kumar S, Goyal SM, Appert J, et al. Association of Airborne Virus Infectivity and Survivability with its Carrier Particle Size. Aerosol Sci Technol. 2013;47(4):373-82. doi: 10.1080/02786826.2012.754841.

20. Alonso C, Raynor PC, Davies PR, Torremorell M. Concentration, Size Distribution, and Infectivity of Airborne Particles Carrying Swine Viruses. PLoS One. 2015;10(8):e0135675. doi: 10.1371/journal.pone.0135675.

21. Lednicky JA, Lauzardo M, Fan ZH, Jutla A, Tilly TB, Gangwar M, et al. Viable SARS-CoV-2 in the air of a hospital room with COVID-19 patients. Int J Infect Dis. 2020;100:476-482. doi: 10.1016/j.ijid.2020.09.025.

22. Scott GH, Sydiskis RJ. Responses of mice immunized with influenza virus by serosol and parenteral routes. Infect Immun. 1976;13(3):696-703. doi: 10.1128/iai.13.3.696-703.1976. 
23. Xing Y-F, Xu Y-H, Shi M-H, Lian Y-X. The impact of PM2.5 on the human respiratory system. J Thorac Dis. 2016;8(1):E69-74. doi: 10.3978/j. issn.2072-1439.2016.01.19.

24. Brooks JT, Beezhold DH, Noti JD, Coyle JP, Derk RC, Blachere FM, et al. Maximizing Fit for Cloth and Medical Procedure Masks to Improve Performance and Reduce SARS-CoV-2 Transmission and Exposure, 2021. MMWR Morb Mortal Wkly Rep. 2021;70(7):254-257. doi: 10.15585/ mmwr.mm7007el.
25. O’Kelly E, Arora A, Pirog S, Pearson C, Ward J, Clarkson PJ. Face Mask Fit Hacks: Improving the Fit of KN95 Masks and Surgical Masks with Fit Alteration Techniques. medRxiv 2020. 10.28.20221895; doi: 10.1101/2020.10.28.20221895.

26. Centers for Disease Control and Prevention. COVID-19 and Your Health [Internet]. CDC; 2020 [citado 27 de agosto de 2021]. Disponible en: https://www.cdc.gov/coronavirus/2019-ncov/your-health/effective-masks.html. 